\title{
Inelastic Buckling of Concrete Column in Braced Frame
}

\author{
By Zdeněk P. Bažant, ${ }^{1}$ Fellow, ASCE, and Yuyin Xiang ${ }^{2}$
}

\begin{abstract}
The paper proposes an improved method of analysis of reinforced concrete columns in braced (no-sway) frames, which is suitable as a simple computer solution for design practice and is more realistic than the existing ACI and CEB methods. The elastic restraint provided by beams adjacent to columns is described by rotational springs. The inelastic behavior of concrete is defined by a uniaxial stress-strain curve with postpeak softening in compression and a zero strength in tension. Plasticity of reinforcement is also considered. The deflection curve is assumed to be a sine curve. The improvement consists in considering the wavelength as unknown and variable during loading. The problem is reduced to a system of seven nonlinear algebraic equations, which are easily solved for small increments of axial displacement by a standard library optimization algorithm. The convergence always occurs and is fast if the increments are small enough. The influence of various parameters on the load-deflection curve, the path in the diagram of axial load $P$ versus moment $M$, and the failure envelope are studied. Various phenomena, such as the possibility of a concave $P(M)$ path at constant load eccentricity, are explained. It is shown that the ACI approach is slightly conservative in most cases, although situations exist in which the ACI approach is either grossly overconservative or slightly unconservative.
\end{abstract}

\section{INTRODUCTION}

Although much has been learned about the inelastic analysis of reinforced concrete columns [e.g., MacGregor et al. (1970); McGregor (1986); Bažant and Cedolin (1991), Sec. 11.5], the existing simple methods still do not ensure a uniform safety margin against collapse. More accurate results can be obtained with geometrically nonlinear inelastic finite element codes. But such an approach is unnecessarily sophisticated and too cumbersome for regular practice, and it does not directly reveal the phenomena during collapse and does not provide understanding of the essential mechanism of failure.

The objective of the present paper is to propose an improved method of analysis of columns in braced frames that considers more degrees of freedom than the existing methods. The method is nevertheless simple enough for use as an easily programmed computer solution in design practice, although not as simple as the existing approach in ACI (American Concrete Institute) Standard 318. The method is demonstrated by numerical solutions and the influences of various parameters as well as diverse phenomena during collapse are studied.

The existing simple methods generally either assume or imply the deflection curve of the column to be sinusoidal (Broms and Viest 1958a,b,c; McGregor et al. 1970; Furlong 1983, 1993; "Building" 1989; "CEB-FIP" 1988). A major assumption in the $\mathrm{ACI}$ code procedure and other current design approaches is that an inelastic column with end restraints can be replaced by an equivalent hinged column of sinusoidal deflection curve. The existing methods also assume the wavelength of the sine curve to be constant during the loading process. This assumption is implied when the effective length (or slenderness) of column is determined by elastic stability analysis, or when the elastically calculated amplification factor is needed [current $\mathrm{ACI}$ and Comité Euro-International de Béton (CEB) design approaches]. But the assumption of constant wavelength is an unnecessary simplification. Numerical solutions by finite elements show that the distribution of curvature during the loading process varies and that the optimum approximation of the deflection curve by a sine curve requires varying the wavelength during the loading process.

\footnotetext{
${ }^{1}$ Walter P. Murphy Professor of Civil Engineering, Northwestern Univ., Evanston, IL 60208.

${ }^{2}$ Grad. Res. Asst., Northwestern Univ., Evanston, IL

Note. Associate Editor: John B. Mander. Discussion open until October 1,1997 . To extend the closing date one month, a written request must be filed with the ASCE Manager of Journals. The manuscript for this paper was submitted for review and possible publication on August 14 1995. This paper is part of the Journal of Structural Engineering, Vol. 123, No. 5, May, 1997. CASCE, ISSN 0733-9445/97/0005-0634-0642 $\$ 4.00+\$ .50$ per page. Paper No. 11392
}

The main improvement proposed in the present paper is to abandon the simplifying assumption of an equivalent hinged column of fixed length and consider the deflection curve to be a sine curve with a wavelength that is variable, is unknown in advance, and is to be solved. A gradual decrease of the wavelength makes it possible to approximately capture the gradual redistribution of curvature during loading, which is the basic characteristic of inelastic response of columns. When the wavelength is shorter than the column length, it is possible to simulate buckling of a column with reverse curvature, which is an important phenomenon in frames. The reverse curvature aspect was recently also studied by Furlong (1993); however, the solution implied the restrictive simplifying assumption that the column ends are either hinged or fixed, rather than restrained elastically, and that the curvature distribution remains the same as in the linear elastic solution.

\section{MATHEMATICAL FORMULATION}

Consider the buckling of interior columns of a large regular reinforced concrete frame in which each story has height $l$ and each bay has span $l_{b}$. For the sake of simplicity, we neglect the differences between the axial forces of the columns of adjacent floors, which permits assuming a regular and periodic buckling pattern (Fig. 1). The frame is perfectly braced, that is, there is no sway. The first buckling mode of the frame has the shape shown in Fig. 1 (Bažant and Cedolin 1991), in which the columns generally exhibit portions of reverse curvature [Fig. 1(b)]. All the columns buckle identically, and therefore the end moments $M_{1}$ of the columns are the same at each joint, and the joint rotations $\theta_{1}$ are also the same although of alternating signs. Likewise, the end moments of the beams $M_{b}$ are the same at all the joints, but of alternating signs. From the equilibrium diagram of the moments acting on each joint (Fig. 1) it follows that $M_{1}=M_{b}$. Furthermore, the primary axial forces in the horizontal beams are zero, and the secondary forces induced by buckling are higher-order small and thus negligible. Therefore, the stiffness of the horizontal beams is constant, independent of the axial forces $P$ in the columns. Noting that the bending moment is uniform in each beam, we deduce from the virtual work principle that $\theta_{1}=M_{1} l_{b} /\left(2 E I_{b}\right)$. Hence, the pair of horizontal beams at each joint is equivalent to a spring of stiffness $C=M_{1} / \theta_{1}$ or $C=2 E I_{b} / l_{b}$.

For the sake of generality, and because it will cause no significant complication of analysis, we consider more generally a column with springs of unequal stiffnesses $C_{1}$ and $C_{2}$ as shown in Fig. 1(b). It must be warned, however, that this cannot represent a frame in which the column stiffness (depending on the axial load) varies from floor to floor. The anal- 

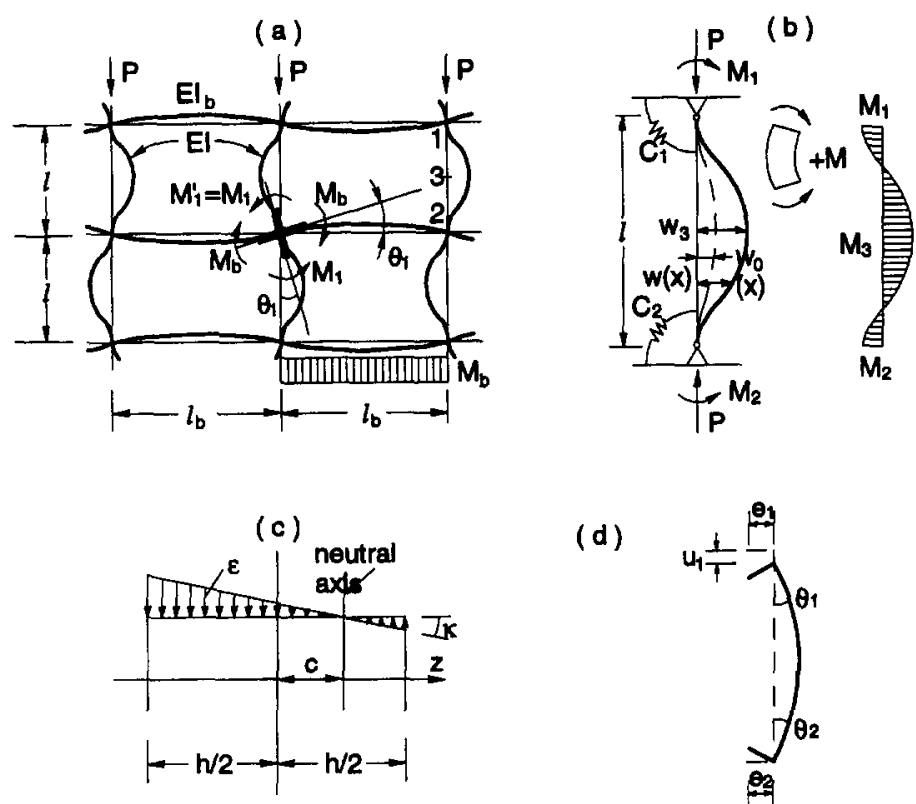

(d)

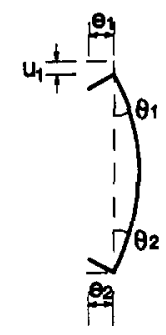

FIG. 1. Deflections and Stralns: (a) Buckling of Group of Interior Members of Regular Frame; (b) Restraints and Buckling of Column In Frame; (c) Strain Dlstrlbution in Cross Section; (d) End Eccentricities and Axlal Displacement

ysis can be reduced to a single column only when the columns in adjacent floors buckle identically as shown in Fig. 1. Otherwise the group of interacting columns would have to be analyzed as a whole, which would be considerably more complicated and would introduce further parameters into the problem.

Let the column be initially subjected to primary moments $M^{0}(x)$ with values $M_{1}^{0}$ and $M_{2}^{0}$ at the top and bottom ends (x = axial coordinate measured from the column top). Subsequently, the axial load $P$ is applied. This results in total lateral deflections $w(x)$ and changes the bending moment to $M(x)$, with values $M_{1}, M_{2}$, and $M_{3}$ at the top, bottom, and midheight of the column, respectively. The equilibrium conditions yield

$$
\begin{aligned}
M_{1} & =M_{1}^{0}+C_{1} \theta_{1} ; \quad M_{2}=M_{2}^{0}+C_{2} \theta_{2} ; \quad M_{3}=\frac{1}{2}\left(M_{1}^{0}+M_{2}^{0}\right. \\
& \left.+C_{1} \theta_{1}+C_{2} \theta_{2}\right)-P w_{3}
\end{aligned}
$$

where $w_{3}=$ midspan deflection.

Even though the column is inelastic, a good approximation can usually be obtained considering the deflection curve $w(x)$ to be the same as for elastic analysis, although with a different wavelength. Thus, we consider

$$
w=A \sin \lambda x+B \cos \lambda x+C x+D
$$

where $A, B, C, D$, and $\lambda$ are unknown constants; $\lambda=\pi / L=$ wavelength parameter ( $L=$ half wavelength). The deflections at the top and bottom ends and at midheight are then expressed as

$$
\begin{gathered}
w_{1}=B+D=0 \\
w_{2}=A \sin \lambda l+B \cos \lambda l+C l+D=0 \\
w_{3}=A \sin \frac{\lambda l}{2}+B \cos \frac{\lambda l}{2}+\frac{C l}{2}+D
\end{gathered}
$$

The slopes and curvatures are calculated as $\theta=w^{\prime}=d w / d x$, $\kappa=w^{\prime \prime}=d^{2} w / d x^{2}$, and their values at top, bottom, and midheight are

$$
\theta_{1}=A \lambda+C ; \quad \theta_{2}=-A \lambda \cos \lambda l+B \lambda \sin \lambda l-C
$$

$$
\begin{gathered}
\kappa_{1}=-B \lambda^{2} ; \quad \kappa_{2}=-(A \sin \lambda l+B \cos \lambda l) \lambda^{2} ; \\
\kappa_{3}=-[A \sin (\lambda l / 2)+B \cos (\lambda l / 2)] \lambda^{2}
\end{gathered}
$$

As usual, the cross sections may be assumed to remain plane and normal to the deflected beam axis, and the reinforcement not to slip against concrete. Then, the axial strain at any coordinate $z$ measured from the column centroidal axis is

$$
\varepsilon=\kappa(z-c)
$$

where $c=$ coordinate of the neutral axis [Fig. 1(c)]

The tensile cracking is taken into account by neglecting the tensile resistance of concrete. This is known to give a very good approximation of the column strength, but, as far as curvatures are concerned, the actual behavior is appreciably stiffer, because of the phenomenon known as tension stiffening (consisting of the tensile resistance of concrete between the cracks). Nevertheless, in buckling, this phenomenon probably is much weaker than in bending of beams, and at the same time there are insufficient data for the role of tension stiffening in buckling. Therefore, the tension stiffening is neglected in the present analysis. Anyway, this simplification is generally on the safe side.

For calculation of the maximum loads, it is necessary to consider the postpeak strain softening of concrete in compression. Although it is known from the theories of damage localization and fracture mechanics that no generally applicable and unique descending postpeak stress-strain relation actually exists, it may be assumed that, at least for the range of practical column sizes and cross-section types, a unique stressstrain relation, $\varepsilon_{c}=f\left(\sigma_{c}\right)$, can be considered ( $\sigma_{c}=$ stress in concrete). A host of formulas could be used. Since ACI does not specify any particular formula, we use the formula recommended in 'CEB-FIP"' (1988)

$$
\begin{gathered}
\text { for } \xi \leq \xi_{u}: \sigma_{c}=f_{c}^{\prime} \frac{m \xi-\xi^{2}}{1+(m-2) \xi} \\
\text { for } \xi>\xi_{u}: \sigma_{c}=f_{c}^{\prime}\left[\left(\frac{n}{\xi_{u}}-\frac{2}{\xi_{u}^{2}}\right) \xi^{2}+\left(\frac{4}{\xi_{n}}-n\right) \xi\right]^{-1}
\end{gathered}
$$

where $f_{c}^{\prime}=$ standard cylinder compression strength of concrete; $\xi=\varepsilon / \varepsilon_{c}^{p}=$ relative strain; $\varepsilon_{c}^{p}=$ strain at peak stress $\approx 0.0022$; $E_{c}^{0}=$ initial modulus $\approx 0.98 \times 10^{4}\left(f_{c}^{\prime} / 0.98\right)^{1 / 3} ; E_{c}^{p}=f_{c}^{\prime} / \varepsilon_{c}^{p} ; \xi_{u}$ $=\varepsilon_{c}^{u} / \varepsilon_{c}^{p} ; \varepsilon_{c}^{u}=$ postpeak strain at $\sigma_{c}^{u}=0.5 f_{c}^{\prime} ; m=E_{c}^{0} / E_{c}^{p} ;$ and $n$ $=4\left[\xi_{u}^{2}(m-2)+2 \xi_{u}-M\right] /\left[\xi_{u}(m-2)+1\right]^{2}$ (all the stresses and moduli are in $\mathrm{MPa}$ ). The relation of stress $\sigma_{s}$ and strain $\varepsilon_{s}$ in steel is assumed to be elastic-perfectly plastic

$$
\text { for } \varepsilon_{s} \leq \varepsilon_{s}^{y}: \sigma_{s}=E_{s}^{0} \varepsilon_{s} ; \quad \text { for } \varepsilon>\varepsilon_{s}^{y}: \sigma_{s}=f_{y}
$$

where $f_{s}^{y}=$ yield stress of steel; $\varepsilon_{s}^{y}=$ strain at the start of yielding; and $E_{s}^{0}=$ elastic modulus of steel. Unloading is assumed to be elastic for both steel and concrete. Although unloading of concrete after compression loading is more complex, it is unimportant for the present analysis because only small portions of the cross section near the neutral axis ever experience unloading.

From the strains corresponding to a given $c$ and $\kappa$, the stress distribution throughout the cross section can be calculated. Integration provides the resultant bending moment $M$ and axial force $P$ (taken positive for compression). The axial force at column midheight must be equal to that at column top and bottom. The bending moments at top, bottom, and midheight can be expressed in terms of the primary moments, end rotations, midspan deflection, and $P$. This yields the equilibrium relations, which can be written in the form

$$
F_{1}=P\left(c_{1}, \kappa_{1}\right)-P\left(c_{3}, \kappa_{3}\right)=0 ; \quad F_{2}=P\left(c_{2}, \kappa_{2}\right)-P\left(c_{3}, \kappa_{3}\right)=0
$$




$$
\begin{aligned}
& F_{3}=M\left(c_{1}, \kappa_{1}\right)-\left(M_{1}^{0}+C_{1} \theta_{1}\right)=0 ; \quad F_{4}=M\left(c_{2}, \kappa_{2}\right) \\
& -\left(M_{2}^{0}+C_{2} \theta_{2}\right)=0 \\
& F_{3}=M\left(c_{3}, \kappa_{3}\right)-\left[\frac{M_{1}^{0}+M_{2}^{0}}{2}+\frac{C_{1} \theta_{1}+C_{2} \theta_{2}}{2}-P w_{3}\right]=0
\end{aligned}
$$

The left-hand sides of these equations have been denoted as $F_{1}, \ldots, F_{5}$; and $c_{1}, c_{2}, c_{3}, \kappa_{1}, \kappa_{2}$, and $\kappa_{3}$ are the neutral axis coordinates and column curvatures at top, bottom, and midheight. The exact solution of these nonlinear equations is obtained when $F_{1}=\ldots F_{3}=0$, and an approximate solution when $F_{1}, \ldots, F_{s}$ are very small numbers. Further we need an expression for the curvature at midspan, which is

$$
F_{6}=\left[A \sin \frac{\lambda l}{2}+B \cos \frac{\lambda l}{2}\right] \lambda^{2}-\kappa_{3}=0
$$

Substitution of (4) and (5) into (9) and (10) yields, together with (12), a system of six equations for six unknowns: $c_{1}, c_{2}$, $c_{3}, A, B$, and $\lambda$. Constants $C$ and $D$ are assumed to be expressed in terms of $A$ and $B, \lambda$ according to (3).

For the programmer, a very effective way to solve the foregoing system of nonlinear equations is to use the LevenbergMarquardt algorithm for nonlinear optimization, which is available in standard computer library subroutines. No further rearrangement of the foregoing equations is needed for that purpose. One merely needs to program the calculation of the values of functions $F_{1}, \ldots, F_{6}$ from the values of $c_{1}, c_{2}, c_{3}$, $A, B, \lambda$, and $P$ [see Fig. 1(d)].

For stability analysis, one further needs the value of the load-point axial displacement $u_{1}$. It represents the sum of the displacements due to the rotations of the end cross sections and the shortening of the column due to the slope of the deflection curve, that is

$$
u_{1}=\theta_{1} e_{1}+\theta_{2} e_{2}+\int_{0}^{l}\left(\frac{w^{\prime 2}}{2}\right) d x
$$

or

$$
\begin{aligned}
u_{1} & =\theta_{1} e_{1}+\theta_{2} e_{2}+\frac{\lambda^{2} l}{4}\left(A^{2}+B^{2}\right)-\frac{C^{2} l}{2}+\frac{A^{2}-B^{2}}{8} \lambda \sin (2 \lambda l) \\
& -\frac{A B \lambda}{2} \sin ^{2}(\lambda l)
\end{aligned}
$$

To be able to keep the numerical solution stable up to the maximum load, it is necessary to consider the load-point displacement $u_{1}$ as given and the axial load $P$ as unknown. Substitution of (4) and (5) into (9)-(12) yields, together with (14), a system of seven nonlinear equations for the unknowns $c_{1}$, $c_{2}, c_{3}, A, B, \lambda$, and $P$.

The subroutine of the Levenberg-Marquardt algorithm automatically varies the values of $c_{1}, c_{2}, c_{3}, A, B, \lambda$, and $P$ so as to minimize the following sum:

$$
S=\sum_{i=1}^{7} F_{i}^{2}=\min
$$

When $S=0$, one obviously has $F_{i}=0$ for all $i=1, \ldots, 7$, that is, the exact solution. In practice, the subroutine converges to a small but positive value of $S$, and if this value is less than a certain prescribed tolerance, one has an acceptable approximate solution. The subroutine generates internally the values of the tangential stiffness matrix of the equation system with respect to the unknowns, but the programmer does not need to bother with this.

Because the equations are highly nonlinear, the objective function $S$ typically has many local minima. So, starting from arbitrary initial values, the algorithm would be likely to converge to an incorrect local minimum. The problem can be avoided as follows. One starts from a case for which the solution is known. This is the case of a very small axial load, for which the solution is easily obtained by elastic analysis (no cracking in concrete) with no nonlinear geometric effects. Then the load-point displacement $u_{1}$ is gradually incremented. For each value of $u_{1}$, the initial values for the nonlinear optimization algorithm are taken as the solution for the previous value of $u_{1}$, which are very close if the change of $u_{1}$ from one solution to the next is chosen sufficiently small. This way one traces the load-deflection curve of the column, which is in fact the objective of the analysis.

For structures under a single dead load or a load system with a single parameter $P$, the limit of stability is reached when

$$
\frac{d P}{d u_{1}}=0
$$

This represents the failure condition. Usually, this condition is equivalent to

$$
\frac{d P}{d M}=0
$$

In certain circumstances, however, the latter condition may be incorrect, as we point out later.

\section{NUMERICAL STUDIES}

Consider now columns of square cross sections, as shown in Fig. 2. The column slenderness is defined as $l / r$, where $r=$ $h / \sqrt{12}$ and $h=$ side of the square cross section $=560 \mathrm{~mm}$. In numerical calculations, we consider $f_{c}^{\prime}=34.48 \mathrm{MPa}$. The column is reinforced symmetrically by four axial steel bars and the steel ratio $\rho_{s}=0.03$. The cover of concrete bars is such that the axial bar centers are $75 \mathrm{~mm}$ from the surface. Furthermore, $E_{s}=2 \times 10^{5} \mathrm{MPa}$ and $f_{y}=414 \mathrm{MPa}$.

First, one must decide the loading path by which the axial force $P$ and bending moment $M$ are increased to their ultimate values $P_{u}$ and $M_{u}$. In this regard it may be noted that the actual loading process that the structure might experience need not be followed in calculations because the response is almost independent on the loading path (and is in fact completely pathindependent as long as no cracks in the cross sections are closing during any part of the loading process). One possible loading path (used in the CEB model column method) is to apply first the axial load $P$ and then increase the bending moment $M$ at constant $P$ until failure is reached. Another way is to increase $P$ and $M$ proportionally, in which case the load eccentricity $e=M / P$ is constant. We consider this second type of loading path. Because the column is buckling, the axial load eccentricity is kept constant only at the column ends, while at all other cross sections the load eccentricity varies.

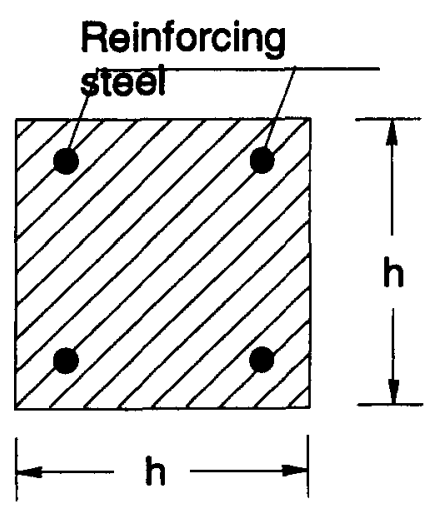

FIG. 2. Cross Section of Reinforced Concrete Column 
The forces and moments plotted in Figs. 3-12 have been normalized to make them dimensionless: $P^{\prime}=P / f_{c}^{\prime} A_{g}, P_{t}^{\prime}=$ $P_{l} / f_{c}^{\prime} A_{g}, P_{r}^{\prime}=P_{r} / f_{c}^{\prime} A_{g}, P_{0}^{\prime}=P_{0} / f_{c}^{\prime} A_{g}, M^{\prime}=M / f_{c}^{\prime} A_{g} h$, and $M_{0}^{\prime}=M_{0} / f_{c}^{\prime} A_{g} h$.

\section{Isolated PIn-Ended Columns (No Springs)}

Since the load eccentricities $e_{1}$ and $e_{2}$ at the column ends are considered constant, the moments applied at the column ends are $M_{1}^{0}=P e_{1}$ and $M_{2}^{0}=P e_{2}$. First, we consider a simply supported column, in which case there are no springs at the
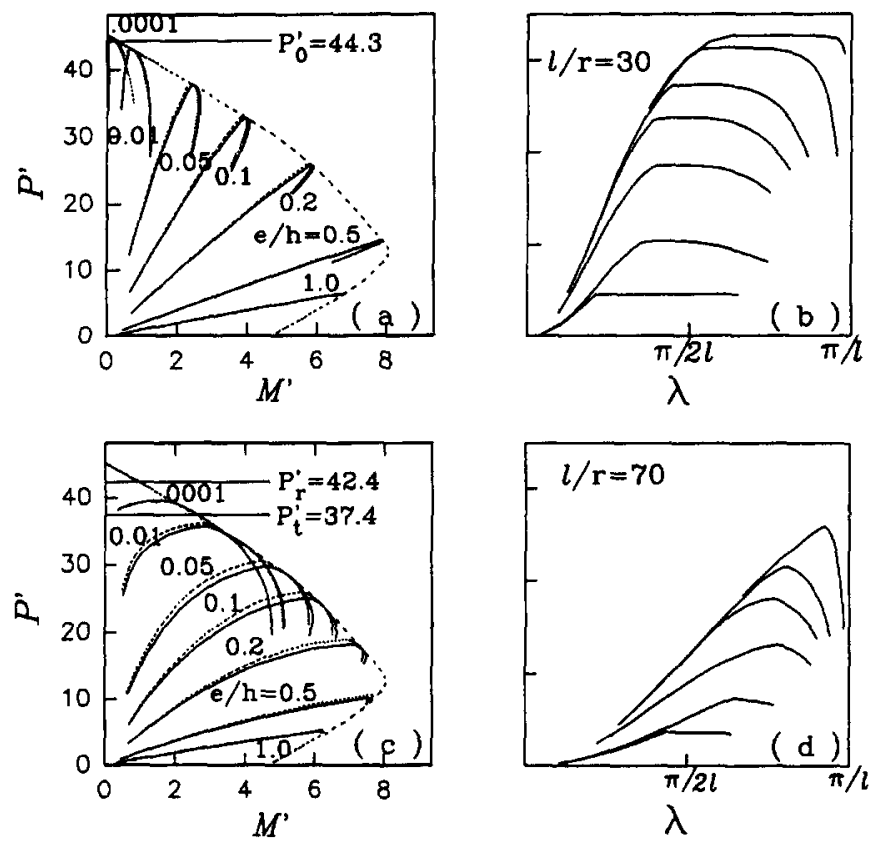

$\lambda$
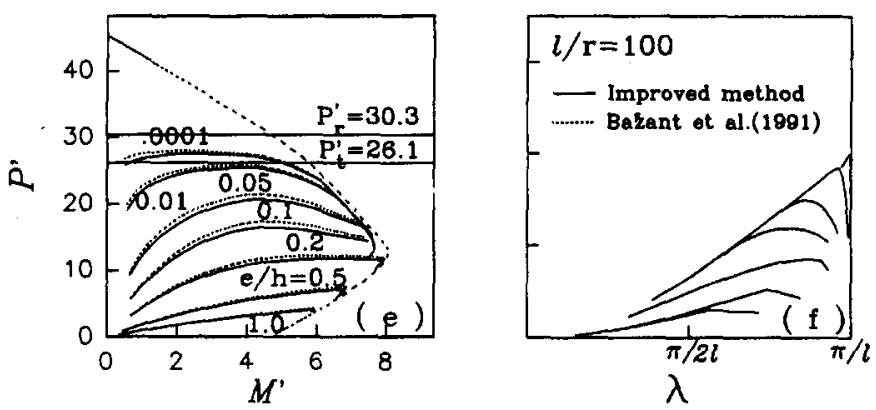

FIG. 3. Response Curves at Increasing Load $P$ for Constant Load Eccentricity e end, or $C_{1}=C_{2}=0$. Figs. 3(a,b) show the results of the aforementioned solution procedure for small slenderness $l / r=30$, Figs. 3(c,d) for medium slenderness $l / r=70$, and Figs. 3(e,f) for high slenderness $l / r=100$.

Fig. 3 also compares the results to the solution according to the simplified method proposed by Bažant et al. (1991), which is similar to the present method except that the wavelength characterized by $\lambda$ is not determined by calculations. Rather, the column is assumed to be replaced by an equivalent hinged column deflecting in the form of a full half sine wave of a fixed length. This has been a customary simplification [e.g., Broms and Viest $(1958 \mathrm{a}, \mathrm{b}, \mathrm{c})]$, used, for example, in the CEB model column method. A half wave sinusoidal shape is also implied in the use of the amplification factor $\left(1-P / P_{c r}\right)^{-1}$ in ACI Specification ['Building”' (1989); “CEB-FIB'” (1988)], because this factor is derived from (and is valid only for) a sinusoidal deflection curve. It is seen that the error of the simplified method compared to the present method is relatively small, especially when the slenderness $l / r$ is not too high. When the slenderness is very high, the differences in the maximum load $P_{\max }$ reach about $5 \%$. Generally, it is seen (Fig. 3) that the present improved method with nonzero curvatures of the deflection curve at the column ends yields a lower $P_{\max }$. This should of course be expected because the present solution considers more degrees of freedom. Figs. 3(b,d,f) further show the evolution of the wavelength parameter $\lambda$ with the axial load $P$. It is interesting to observe that $\lambda$ increases during the loading (or the half wavelength decreases) and approaches $\pi /$ $l$ during collapse (also, $A$ increases with increasing curvature $\kappa$ while $B, C$, and $D$ decrease). Thus the column tends to collapse nearly in the form of a half sine wave, with its effective length $L$ approaching the actual length $l$ (although before collapse $L>l$ ).

For comparison, Fig. 3 further shows Shanley's tangent modulus load $P_{1}$ and Engesser-von Kármán's reduced modulus (or double modulus) load $P_{r}$ [see Bažant and Cedolin (1991), Chapters 8 and 10).

Fig. 4 shows the results of the present method for the case that the end moments are first applied at zero axial load and are then kept constant while the axial load is increased up to failure. The paths of axial load $P$ versus maximum bending moment $M$ look quite different from those at constant $e$ (Fig. 3 ), being initially much steeper. The failure envelope $P(M)$ is lower than that obtained at constant $e$ but the difference (caused by irreversible strains at unloading near the neutral axis) is too small to be discernible.

According to the CEB model column method ("CEB-FIP" 1988), the axial load $P$ is applied first at $M=0$, and then $M$ is increased at constant $P$ until failure (which occurs at max-
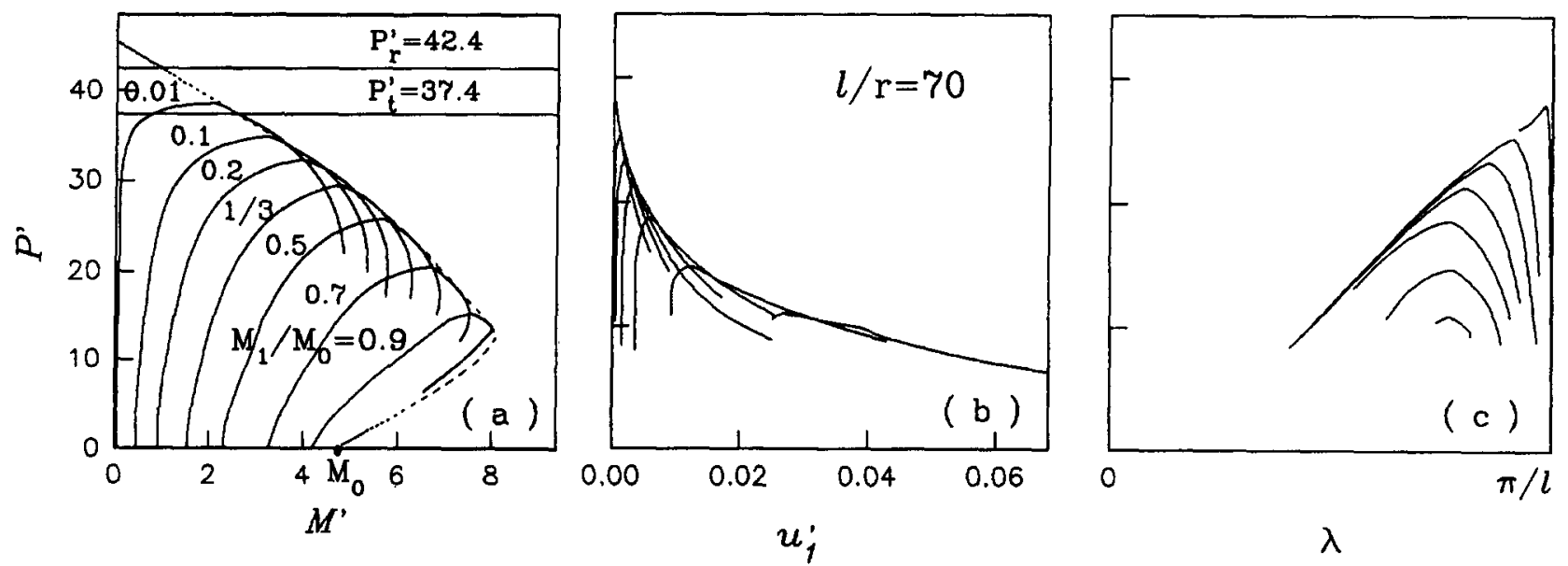

FIG. 4. Response Curves at Increasing Load $P$ for Constant End Moments $M_{1}$ and $M_{2}$ 

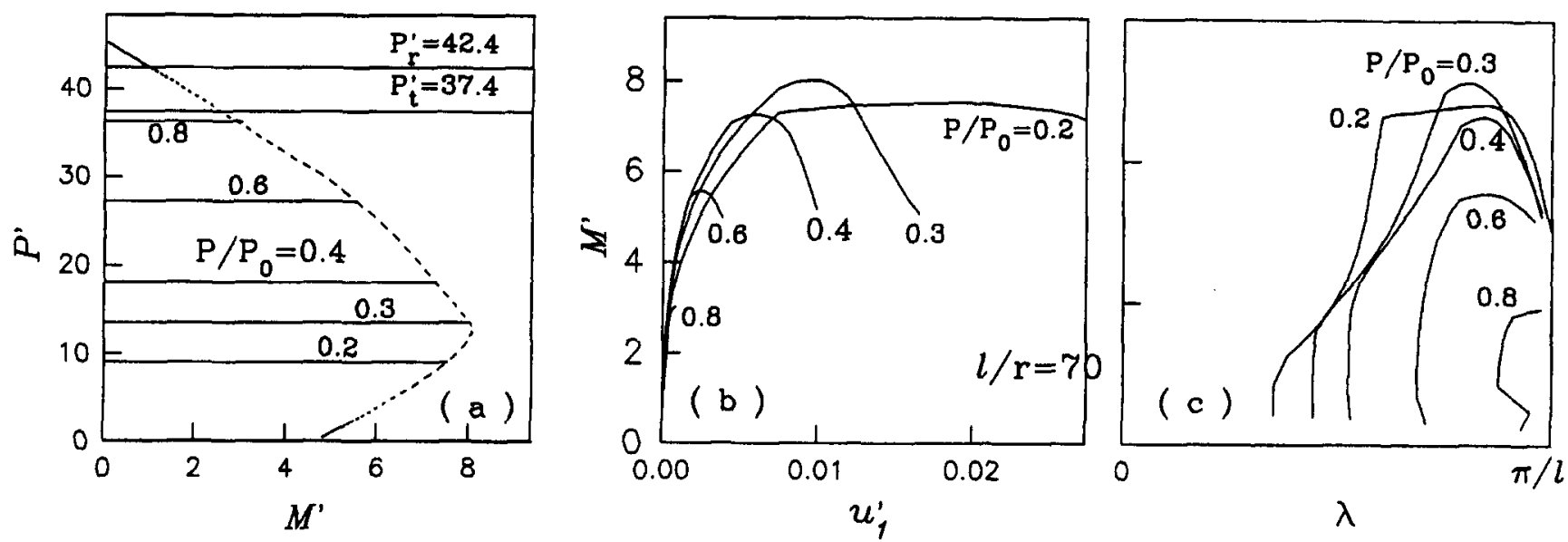

FIG. 5. Response Curves for Increasing Moment $M$ at Constant Load P (Assumed in CEB Model Column Method)
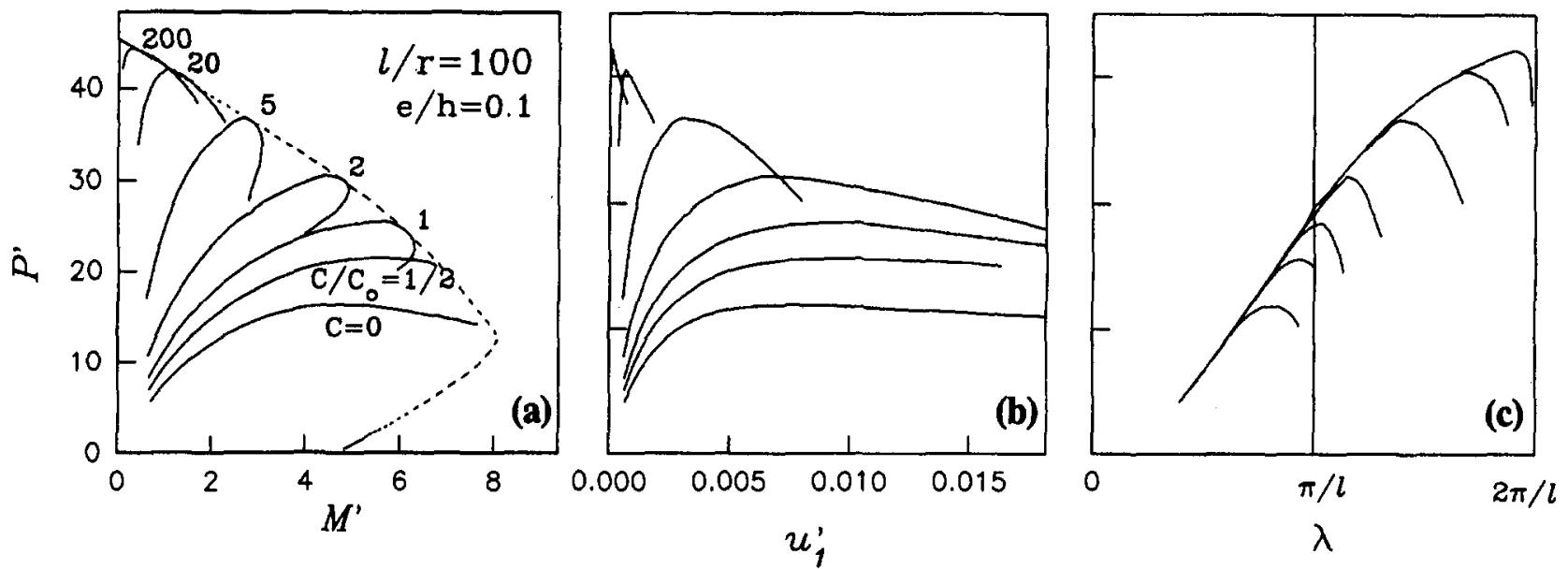

FIG. 6. Response Curves at Increasing Load $P$ for Constant End Eccentrlcity $\theta=0.1 \mathrm{~h}$ and Slenderness $/ / r=100$
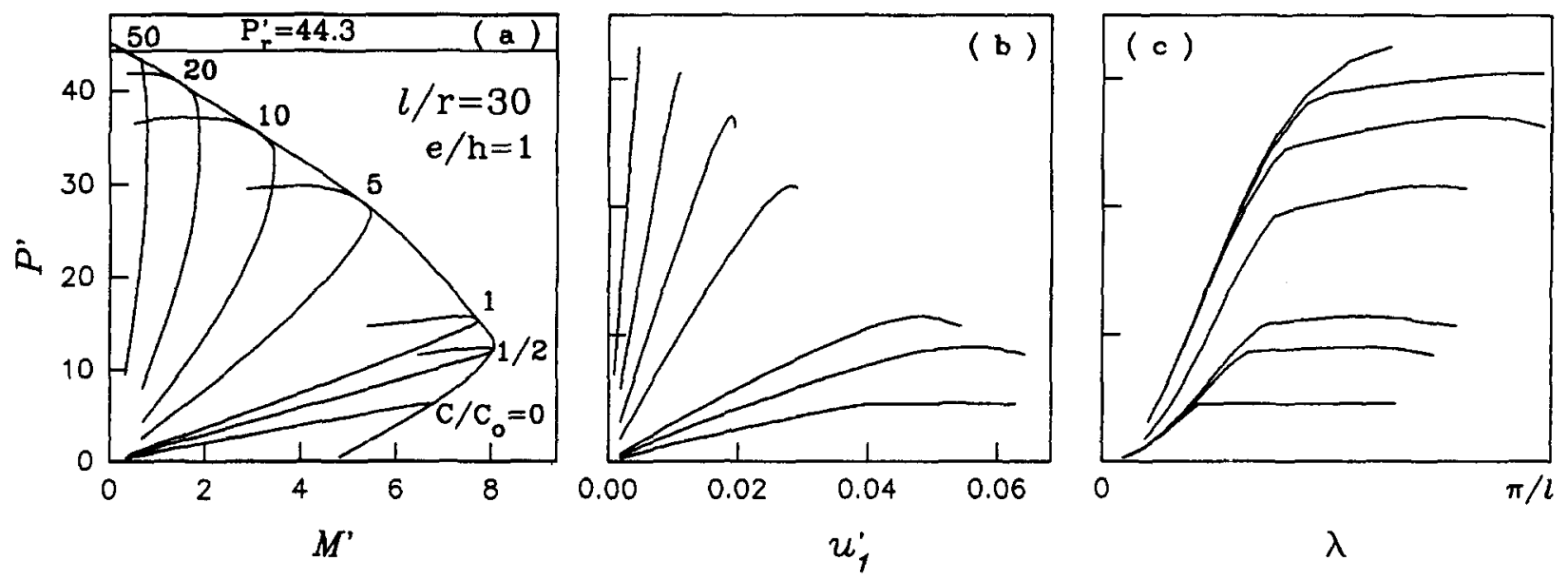

FIG. 7. Reeponse Curves at Increasing Load $P$ for Constant End Eccentriclty $\theta=h$ and Slenderness $/ / r=30$

imum $M$ ). The response for this loading history has been calculated with the present method for a column without springs. The results are plotted in Fig. 5. The failure envelope obtained for loading at constant end eccentricities $e$ is also shown in the figure. As we see again, the dependence of the failure envelope on the load history is very small. The envelope for loading at constant $P$ (used in the CEB method) lies always higher (and thus gives a slightly lower safety margin), but the difference is negligible.

\section{Columns in Frame Modeled by Springs at Ends}

The results of calculations with the present method are shown in Figs. 6-8, in which the spring stiffnesses $C_{1}$ and $C_{2}$ are normalized with respect to the value $C_{0}=E I / l=\left(E_{c}^{0} I_{c}+\right.$ $\left.E_{s} I_{s}\right) / l$ where $I_{s}, I_{c}=$ centroidal moments of inertia of the steel part and the concrete part of the cross section (including the area of cracked concrete), and $E_{s}, E_{c}^{0}=$ elastic moduli of steel and concrete, with superscript 0 referring to the initial value. 

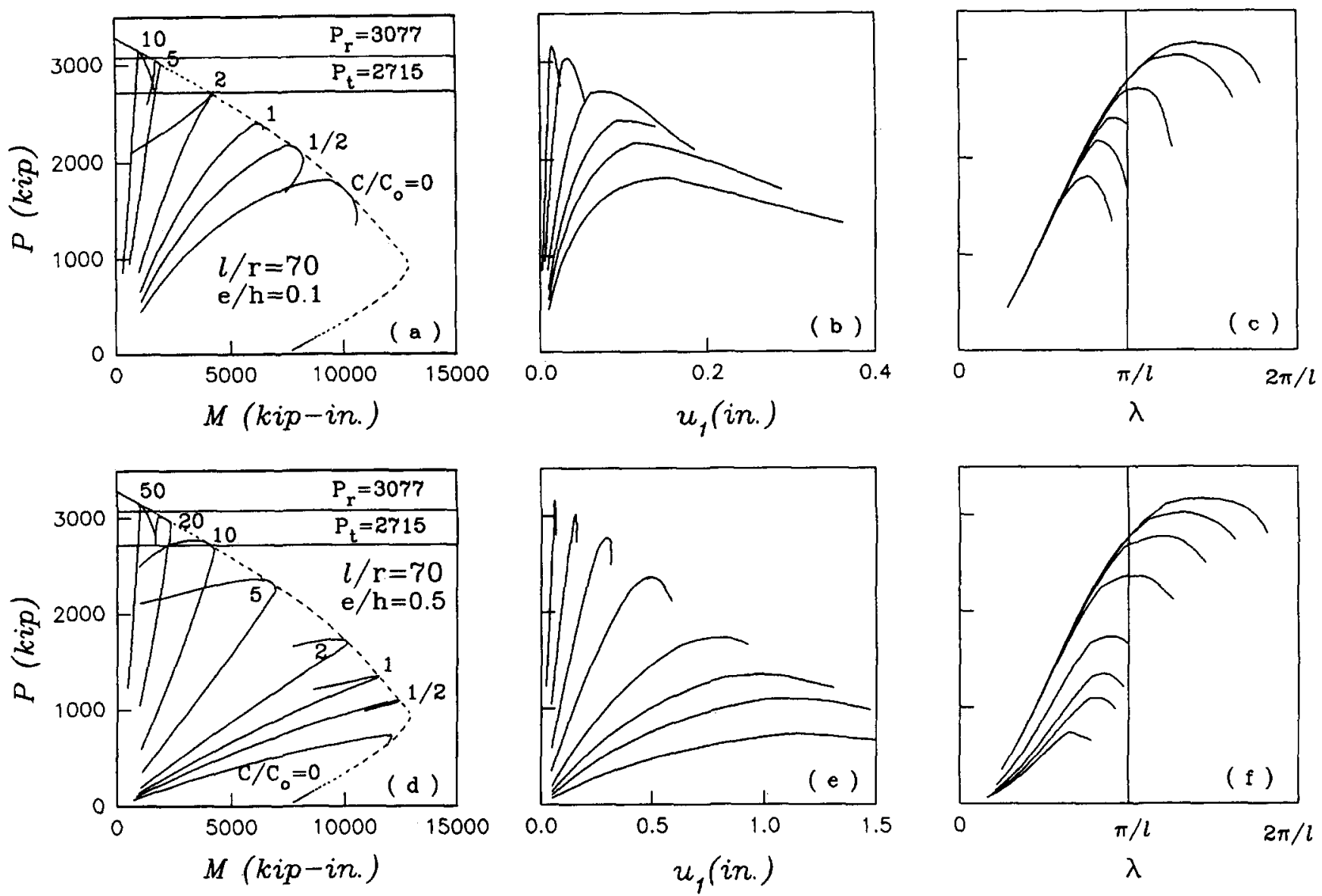

FIG. 8. Response Curves at Increasing Load $P$ for Two Constant End Eccentrlcitles $\theta=0.1 h$ and $0.5 h$, and Slenderness $/ / r=70$
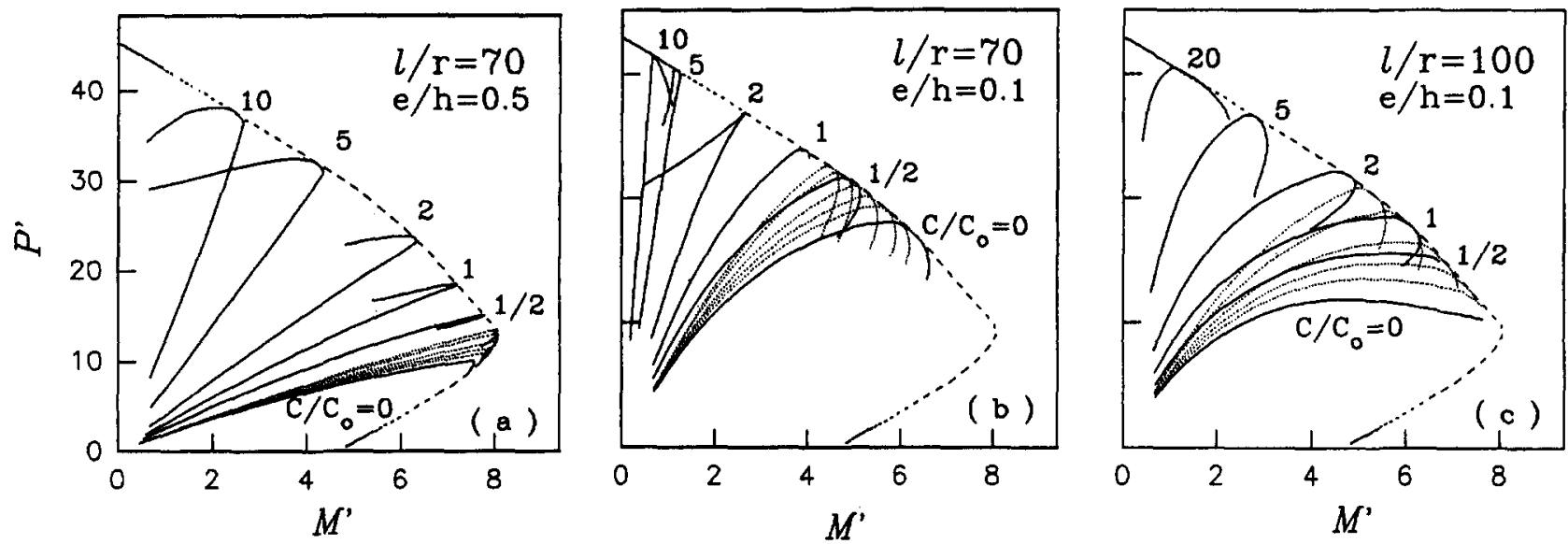

FIG. 9. Response Curves for Elastically Equlvalent Column at Increasing Load P and Various Eccentricitles and Slendernesses

Only the case of equal stiffnesses, $C_{1}=C_{2}=C$, is considered in the figures. The results are calculated for various slendernesses and relative eccentricities, that is, $l / r=100$ with $e / h=$ $0.1 ; l / r=30$ with $e / h=1 ;$ and $l / r=70$ with $e / h=0.1$ and 0.5 . The figures show not only the calculated $P(M)$ diagrams, but also the diagrams of load $P$ versus the axial load-point displacement $u_{1}$. The peak points of these diagrams represent the limits of stability, that is, the failures. Also shown is the evolution of $\lambda$, which is seen to increase during loading $(\lambda=$ $\pi / L$, where $L=$ half wavelength).

As expected, the spring stiffness is seen to have a dramatic effect on the failure load (Fig. 6). We also see that, in contrast to the column with no springs, $\lambda$ can be larger than $\pi / l$ and can even approach $2 \pi / l$. Of course, this is to be expected because the case $C \rightarrow \infty$ corresponds to the fixed-end column.
Fig. 9 shows calculations by the present method when the column with springs at ends is replaced by an elastically equivalent column, defined as a column with the same cross section and no springs at the ends but with a reduced length (effective length) that is equal to the half wavelength of the perfect column at the critical load, calculated according to the theory of elasticity. So far, practical design has typically been based on such an elastically equivalent pin-ended column. The dotted lines are the results from the analysis of the equivalent column. We can observe from the comparison that the equivalent column method is generally very conservative (yielding a smaller critical load), especially in the cases when $l / r$ is small or when $C / C_{0}$ is large. The reason is that when a column has strong springs and a relatively small slenderness, the column is markedly strengthened (as is discussed in more detail later). 

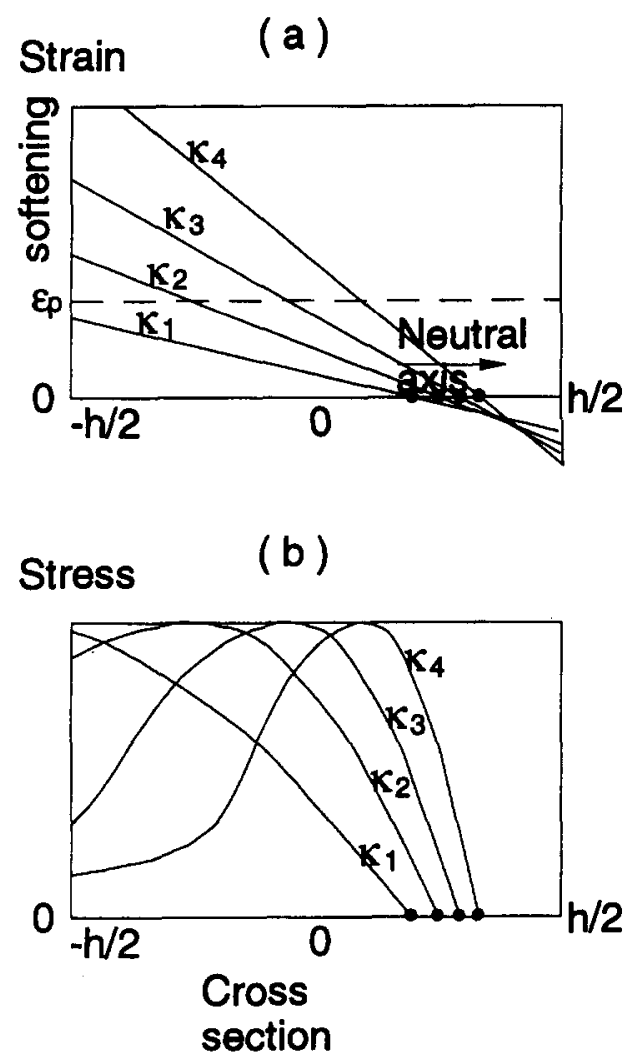

FIG. 10. Typical Subsequent Stress Distributions in Reinforced Concrete Cross Section

But the replacement by an equivalent column without springs diminishes this effect and thus makes the equivalent method much more conservative, except for columns with large slenderness and weak springs (or, weak connected beams) at ends.

For pin-ended columns (so springs), the $P(M)$ diagrams at constant end eccentricities $e$ are always concave (concave curvatures are those shown in Fig. 3). The stability condition can then always be reduced to $d P / d M>0$; that is, the failure corresponds to the peak point of the $P(M)$ diagram. However, for columns with strong enough springs and a relatively small slenderness, the $P(M)$ diagram can be convex.

If $P(M)$ is convex [such as curve 5 in Fig. 8(d)], the condition $d P / d M=0$ in (17) is not the limit of stability, that is, the failure state. Rather, one must use the basic condition of stability, which is $d P / d u_{1}=0[(16)]$. The slope $d P / d M$ becomes negative because the moment decreases $(d M<0)$. But the axial load $P$ does not decrease, and so the column does not lose stability even though $d P / d M<0$.

The physical origin of such behavior is the compressive strain softening of concrete coupled with a high spring stiffness. The convex $P(M)$ diagram occurs when the neutral axis shifts into the cracked portion of the cross section, that is, the compressed area expands [Fig. 10(a)], while at the same time the peak of the compressive stress profile shifts toward the neutral axis and the stress at the compressed face decreases due to strain softening [Fig. 10(b)]. When the strain softening at the compression face is rapid and at the same time the compressed area increases, the axial force can remain approximately constant. However, the arm of the axial force diminishes due to the aforementioned shift of the neutral axis. Consequently, while the bending moment carried by the steel bars remains about the same, the bending moment carried by concrete decreases and causes the total bending moment to decrease, too, at constant axial force. This can produce negative slope $d P / d M$ without a loss of stability. But for both convex and concave $P(M)$ diagrams, the failure under dead loads occurs at the state of maximum load.
Further insight may be gained by noting the equilibrium relation for the maximum moment in the column: $M=P(e+$ $\left.w_{3}\right)-C \theta$, where $w_{3}=$ deflection at the cross section with maximum moment. The end rotation may be estimated as $\theta=$ $\eta w_{3} / l$ (where $\eta$ is a constant, approximately $2-4$ ). Thus, $M / P$ $=e+w_{3}[1-(\eta C / P l)]$. Now, note that a convex $M(P)$ diagram is obtained when $M / P$ decreases at increasing $P$. Such behavior is promoted when stiffness $C$ is large and column length $l$ is small. These are the cases for which the concave $P(M)$ diagrams have been obtained in Figs. 7 and 8(d). However, when the spring stiffness $C$ is extremely high, the column behaves almost as a fixed-end column, which is equivalent to a pinended column of half the length, and so the $P(M)$ diagram is concave again. The transition from a concave to a convex diagram occurs at a certain critical value of the stiffness ratio $C / C_{0}$.

The convex $P(M)$ diagram was experimentally observed by Furlong and Fergusson (1966). But they detected this behavior only for the column ends. For the behavior of the maximum moment cross section, a convex diagram was obtained in an analysis of columns in braced frames by Cranston (1972). He assumed the failure to occur at the point where the convex $P(M)$ curve reaches the envelope of the $P(M)$ curves. This is not the failure state, however, because the column does not become unstable until the point of horizontal tangent is reached.

\section{Comparison with ACI Method}

In the ACI method, the column is designed for magnified maximum moment $M_{\max }=\mu M_{\max }^{0}$, in which $M_{\max }^{0}=$ primary moment (calculated without the second-order geometric effects) and $\mu=C_{m} /\left[1-\left(P / \phi P_{c r}\right)\right]=$ magnification factor, $P_{c r}$ $=\overline{E I} \pi^{2} / L^{2}, \overline{E I}=$ effective bending stiffness of the column cross section, $L=k l=$ effective column length (length of a supposedly equivalent hinged column), which is assumed to be constant during the loading process, $k=$ factor determined by elastic buckling analysis of the column with the proper end restraints, $\phi=$ strength reduction factor, and $C_{m}=$ coefficient introducing the effect of the distribution of the primary (initial) bending moment between the column ends, taken by ACI as $C_{m}=1$. For the purpose of comparison, we consider the case $\phi=1$ (MacGregor 1986; Bažant and Cedolin 1991; Furlong 1983). Furthermore, $\overline{E I}=\alpha E_{c}^{0} I_{c}^{8}+E_{s}^{0} I_{s}$, where $\alpha=0.2$ and $I_{c}^{g}=$ moment of inertia of the gross concrete cross section. The long-time creep effects are not included in the preceding expressions and are neglected. The primary bending moment corresponding to the current ACI method is $M_{\max }^{0}=M_{\max } / \mu$ where $M_{\max }=$ the maximum bending moment calculated with the present method. On the other hand, the first-order analysis of

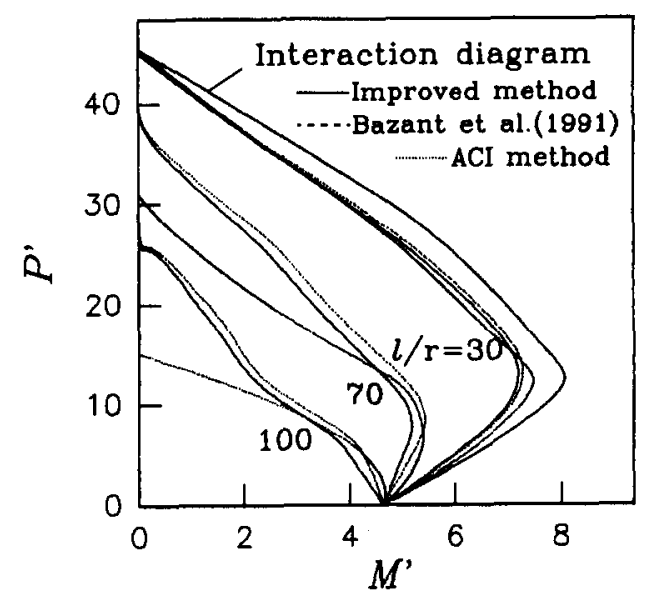

FIG. 11. Failure Envelope for Pin-Ended Columns 

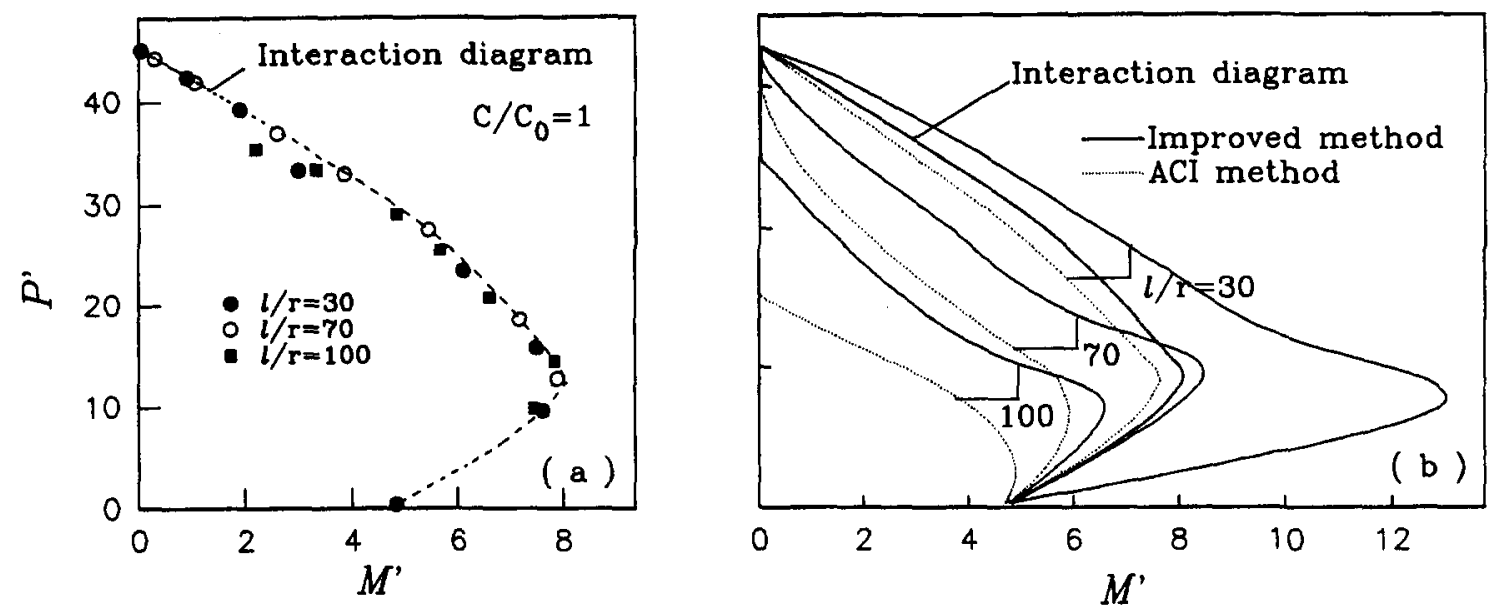

FIG. 12. Failure Envelopes for Columns with Slendernesses $I / r=30,70,100$, and with Spring Stifiness $C=C_{0}$

(a)

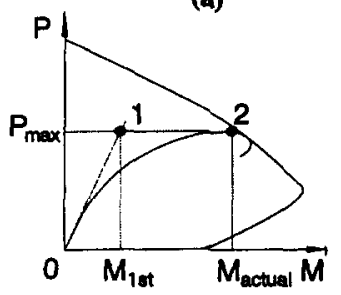

(b)

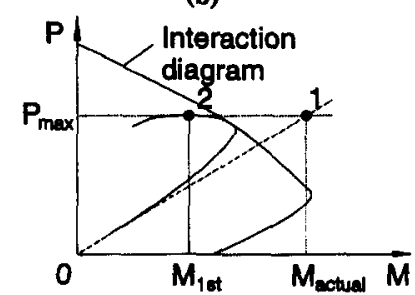

FIG. 13. Different Response Curves at Increasing Load $P$ and Constant Eccentricity e for (a) Stocky; and (b) Slender Columns

the column with springs at the ends yields $\theta=P e /\left(C+2 C_{0}\right)$ and

$$
M_{\max }^{0}=P e /\left(1+C / 2 C_{0}\right)
$$

Figs. 11 and 12 (a) show the failure envelopes of columns with no springs or with symmetric springs at ends. The points in Fig. 12(a) correspond to the maximum points 2 in Fig. 13(b). Figs. 11 and 12(b) show also the diagrams of the corresponding bending moments according to (18). As we see, end springs can increase the column strength greatly. The failure envelopes of columns with spring constants at both ends are very close to the cross section interaction diagram [Fig. 12(a)]. From Fig. 11 we see that the envelopes for springs at ends are very close to those obtained by Bažant et al. (1991), although somewhat lower. However, when the end eccentricity is small and the column is slender, there is a great difference between the ACI envelopes and the present method. In most cases, the ACI method is conservative. But in some cases, it is very conservative, and so it does not achieve the objective of a uniform safety margin. When $e / h$ is large, the ACI method appears to be slightly on the unsafe side.

Fig. 12(b) compares the failure envelopes obtained by the ACI method and the present method. Some envelopes in Fig. 12(b) lie outside the cross section interaction diagram. This response corresponds to large eccentricities and small slendernesses, or to a convex $P(M)$ diagram.

If the $P(M)$ curve is convex, point 1 in Fig. 13 may lie outside the interaction diagram. This is because the slenderness makes the actual moments smaller than the first-order moment. Or, we can say, the slenderness brings $M_{\max }^{0}$ back into the safe domain (inside the interaction diagram).

Note also that the term "reduced failure envelope" for the failure envelope plotted in terms of the primary (first-order) moment is not very appropriate because in some cases the primary (first-order) diagram lies higher than the actual one.

Fig. 12(b) compares the ACI method with the present method for columns having springs at both ends. We see that

the ACI method is generally very conservative, especially for a convex $P(M)$ curve. The ACI method can never yield a diagram outside the interaction envelope (because the magnification factor is never less than 1 ).

Overall it may be observed that the columns with springs at the ends, which replace the effect of the restraints by horizontal beams, are designed according to the ACI method very conservatively [Fig. 12(b)].

Because a convex $P(M)$ curve occurs when there are strong springs at the ends, a large load eccentricity, and a relatively small slenderness, it follows from the present analysis that the ACI method is very conservative for stocky columns with large load eccentricities and strong elastic restraints at ends.

One may wonder how much is the error due to restricting the deflection curve to be sinusoidal. In this regard, it is interesting to note that nearly identical results would be obtained if the deflection curve were considered to be a fourth degree polynomial in $x$ rather than a sine curve.

The foregoing solution can be simplified for symmetric columns $\left(e_{1}=e_{2}, C_{1}=C_{2}\right)$. It reduces to a system of only three nonlinear equations. Of course, the results are exactly the same.

\section{CONCLUSIONS}

1. An effective approach to the analysis of reinforced concrete columns is to assume the deflection curve to be a sine curve whose wavelength is unknown, can vary, and is one of variables to be solved. This novel feature of the present solution represents abandonment of the assumption of an equivalent elastic column of fixed length, used in the existing design approaches. The solution of a column within a no-sway frame, analyzed as a column with arbitrary load eccentricities and arbitrary spring supports at ends, can then be reduced to a system of seven simultaneous nonlinear equations for seven unknowns. These can be effectively solved by a standard computer library algorithm for nonlinear optimization. The axial displacement is incremented in small steps and the converged solution for one load level is used as the initial estimate for the optimization procedure at the next load level. For sufficiently small axial displacement increments, the solution converges, and does so rapidly.

2. The proposed method can capture gradual redistribution of curvature during buckling and can model columns whose deflection curve has portions of reverse curvatures. The method of solution is suitable for design practice when a more realistic solution with lesser simplifi- 
cations than those used in the ACI or CEB codes is desired.

3. Comparisons with calculations according to the current ACI standard 318 show that the ACI approach is conservative in most cases, although there are certain situations with stiff elastic end restraints in which the method is either strongly conservative or slightly unconservative.

4. The calculations also confirm the previously observed fact that the diagram of axial load versus bending moment at constant load eccentricity is not always concave but can be convex, and explain when this happens.

\section{ACKNOWLEDGMENT}

Partial financial support from the ACBM Center at Northwestern University is gratefully appreciated.

\section{APPENDIX. REFERENCES}

Bažant, Z. P., and Cedolin, L. (1991). Stability of structures. Oxford University Press, New York, N.Y.

Bažant, Z. P., Cedolin, L., and Tabarra, M. R. (1991). "New method of analysis for slender columns."' ACl Struct. J., 88(4), 391-401.

Broms, B., and Viest, I. M. (1958a). "Ultimate strength of long hinged reinforced concrete columns." J. Struct. Div., ASCE, 84(1), 15101538.

Broms, B., and Viest, I. M. (1958b). "Ultimate strength analysis of long restrained reinforced concrete columns." J. Struct. Div., ASCE, 84(3), $1635-1650$.

Broms, B., and Viest, I. M. (1958c). "Design of long reinforced concrete columns." J. Struct. Div., ASCE, 84(4), 1694-1728.

"Building code requirements for reinforced concretes and commentary." (1989). ACI 318-89, 318 R-89, American Concrete Institute (ACI), Detroit, Mich.

"CEB-FIP model code." (1988). Bulletin d'Information, No. 190a. Comité Euro-International de Béton, Lausanne, Switzerland.

Cranston, W. B. (1972). "Analysis and design of reinforced concrete columns." Res. Rep. No. 20, Cement and Concrete Association, London, U.K. (Sec. 8.5).

Furlong, R. W. (1983). "Column rules of ACE, SSLC and LRFD compared." J. Struct. Div, ASCE, 109(10), 2375-2386.

Furlong, R. W. (1993). "Slenderness of columns in braced frames." $J$. Struct. Div., ASCE, 119(11), 3405-3415.

Furlong, R. W., and Ferguson, P. M. (1966). "Tests of frames with columns in single curvature." Symposium on reinforced concrete columns, ACI Publ. SP-13, Detroit, Mich., 55-74.

MacGregor, J. G. (1986). "Stability of reinforced concrete building frames." Concrete framed structures, R. Navayanan, ed., Elsevier, London, U.K., 1-41 (Sec. 8.5).

MacGregor, J. G., Breen, J. E., and Pfrang, E. O. (1970). "Design of slender concrete columns." ACI J., 67(1), 6-28. 